\title{
A Method for Generating Helical Surface of the VPA Hob on a CNC Worm Grinding Machine
}

\author{
Van-The Tran ${ }^{1,2}$, Ruei-Hung Hsu ${ }^{3}$, Shih-Sheng Chen ${ }^{1}$ and $\mathrm{Yu}-\mathrm{Ren} \mathrm{Wu}^{1}$ \\ ${ }^{1}$ Department of Mechanical Engineering, National Central University, 300, Jhongda Rd., Jungli City, Taoyuan 320, Taiwan \\ ${ }^{2}$ Department of Mechanical Engineering, Hung Yen University of Technology and Education, 5 Rd., Hung Yen City, Vietnam \\ ${ }^{3}$ Bachelor's Program in Precision System Design, Feng Chia University, 100 Wenhwa Rd., Seatwen, Taichung 40724, Taiwan
}

\begin{abstract}
Variable pressure angle (VPA) hob is an important cutting tool for generating the twist-free tooth flanks on a crowned involute helical gear used in the high precision gear boxes. However, an appropriate method for generating the VPA hob surface has not proposed yet. Therefore, this paper presents a worm grinding method for generating the helical surface of VPA hob on a CNC worm grinding machine. A mathematical model of the proposed method is established based on a standard hob by controlling rotational motion of the hob axis in the worm grinding process. A numerical example is implemented to verify the merits of the proposed method.
\end{abstract}

\section{Introduction}

In batch production of small external gears and consideration of more efficient process, the generating grinding method is usually adopted for the hard finishing of gears instead of the form grinding method. The tooth profile of a cylindrical grinding worm equates a rack profile in the normal section. The involute is generated by the continuous and relative rotation motion between grinding worm and work-piece based on the envelope process. Besides, in the generating grinding process, many points of the grinding worm are always in contact with the gear workpiece and the number of contact points change continuously during the tool rotation. Thus the stock removal rate in the generating grinding of gear is very high. In the past researches, the worm grinding process has been applied for some kind of the gears. Therefore, in this study, we proposed a worm grinding method for grinding the VPA hob on a CNC grinding machine.

The basic geometric design, tooth surface envelope theory, processing method and tool design of various worms are published by Litvin and Fuentes [1]. Also, some related literatures about the worm grinding process are published. Wirz [2]-[4] patented a method, tool and device for profiling the grinding worm that applies in the continuous gear grinding. Besides, a process for dressing a single and multiple-grooved worm by a dressing tool is also proposed. Then a grinding worm that can be profiled on the grinding machine is introduced by Thyssen [5]. Jankowski [6], [7] provides a grinding worm with a geometry and face topology to obtain a high time-cutting volume and a tooth face modifications on the micrometer level of the work gear. Stadtfeld and Mundt [8] patented a dressing method for the threaded grinding wheel that can obtain the twist-free tooth flanks of gear workpiece in the gear grinding process. The profile and lead modifications of a helical gear is produced by using the threaded wheel and profile grinding [9]. Klocke et al. [10], [11] introduced a research method for analyzing the gear generating grinding process based on the manufacturing simulation as well as an analogy trial and the cutting forces of grinding are also determined depending on the process parameters. Denkena et al. [12] presented an approach for analyzing the tool engagement in gear grinding and calculating the material removal rate. Ophey and Reimann [13] introduced a thermomechanical model for describing influence on the surface zone in the gear generating grinding process. Recently, Tran et al. [14], [15] proposed a novel hobbing method to free twist tooth flanks of the longitudinal crowned helical gear by using variable pressure angle hob cutter on $\mathrm{CNC}$ hobbing machine. Besides, the topologies, contact bearings and transmission errors of the double-crowned work gear pairs generated by a modified hob in the gearhobbing process are investigated.

A method for grinding a standard hob as a VPA one is proposed by using the grinding worm on a $\mathrm{CNC}$ grinding machine. A mathematical model of grinding process is also established. Numeral results show that the proposed VPA hob surface generated by grinding worm is close to the theoretical VPA hob surface.

\section{Generation of theoretical VPA hob surface}

Basically, the standard hob's profile is the same as the standard helical gear's profile that can be generated by a 
standard rack cutter. To obtain a twist-free tooth flank of helical gears, the pressure angle of standard rack cutter's profile is modified as a variable function in its longitudinal direction, as shown in Fig. 1. Accordingly, the position vector and unit normal vector of the rack cutter's right-hand side profile can be expressed in the coordinate system $S_{r}\left(x_{r}, y_{r}, z_{r}\right)$ as follows:

$$
\begin{aligned}
& \mathbf{r}_{r}\left(u_{1}, v_{1}\right)=\left[x_{r}, y_{r}, z_{r}, 1\right]^{T} \\
& =\left[u_{1} \cos \alpha_{o n}, u_{1}\left(-\sin \alpha_{o n}+b v_{1}\right), v_{1}, 1\right]^{T}, \\
& \mathbf{n}_{r}\left(u_{1}, v_{1}\right)=\left[n_{x_{r}}, n_{y_{r}}, n_{z_{r}}\right]^{T} \\
& =\left[\sin \alpha_{o n}-b v_{1}, \cos \alpha_{o n},-b u_{1} \cos \alpha_{o n}\right]^{T},
\end{aligned}
$$

where $u_{1}$ and $v_{1}$ are the rack cutter surface parameters, $\alpha_{o n}$ is the pressure angle of the rack cutter, and $b$ is VPA coefficient of rack cutter. The dotted profile of the standard rack cutter are determined by setting the coefficient, $b$, to zero, as shown in Fig. 1.

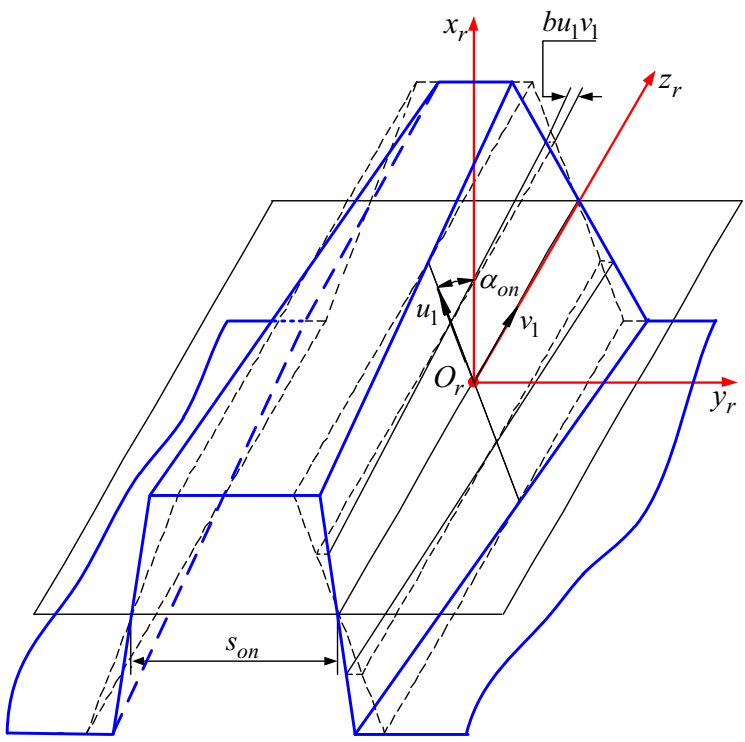

Figure 1. Surface parameters of the VPA rack cutter.

The schematic generation mechanism of the hob is shown in Fig. 2, where coordinate systems $S_{r}\left(x_{r}, y_{r}, z_{r}\right)$, $S_{h}\left(x_{h}, y_{h}, z_{h}\right)$, and $S_{4}\left(x_{4}, y_{4}, z_{4}\right)$ are rigidly connected to the rack cutter, hob, and frame, respectively. By applying the homogenous coordinate transformation matrix equation, the locus and unit normal vectors of the lefthand side rack cutter surface represented in the coordinate system $S_{h}\left(x_{h}, y_{h}, z_{h}\right)$ are attained by

$$
\begin{aligned}
& \mathbf{r}_{h}\left(u_{1}, v_{1}, \varphi_{1}\right)=\mathbf{M}_{h r}\left(\varphi_{1}\right) \cdot \mathbf{r}_{r}\left(u_{1}, v_{1}\right) \\
& =\left[\begin{array}{cccc}
\cos \varphi_{1} & -\cos \beta_{o 1} \sin \varphi_{1} & -\sin \beta_{o 1} \sin \varphi_{1} & r_{o 1}\left(\cos \varphi_{1}+\varphi_{1} \sin \varphi_{1}\right) \\
\sin \varphi_{1} & \cos \beta_{o 1} \cos \varphi_{1} & -\sin \beta_{o 1} \cos \varphi_{1} & r_{o 1}\left(\sin \varphi_{1}+\varphi_{1} \cos \varphi_{1}\right) \\
0 & -\sin \beta_{o 1} & \cos \beta_{o 1} & 0 \\
0 & 0 & 0 & 1
\end{array}\right] \cdot\left[\begin{array}{c}
x_{r} \\
y_{r} \\
z_{r} \\
1
\end{array}\right],
\end{aligned}
$$

$$
\mathbf{n}_{h}\left(u_{1}, v_{1}, \varphi_{1}\right)=\mathbf{L}_{h r}\left(\varphi_{1}\right) \cdot \mathbf{n}_{r}\left(u_{1}, v_{1}\right)
$$

where $\mathbf{L}_{h r}\left(\varphi_{1}\right)$ is the upper-left $(3 \times 3)$ sub-matrix of the $(4 \times 4)$ homogeneous coordinate transformation matrix, $\mathbf{M}_{h r}\left(\varphi_{1}\right)$. And $\varphi_{1}$ is the rotation angle of hob, $\beta_{o 1}$ is the helix angle of rack cutter and $r_{o 1}$ is the operating radius of hob.

According to the theory of gearing, the equation of meshing between the right-hand side rack cutter surface and left-hand side hob surface can be obtained by

$$
f_{1}\left(u_{1}, v_{1}, \varphi_{1}\right)=\mathbf{n}_{h}^{T} \cdot \partial_{\varphi_{1}}\left[x_{h}, y_{h}, z_{h}\right]=0
$$

Then, the locus of tooth profile and its unit normal of the hob are obtained by simultaneously considering Eqs. (3), (4) and (5).

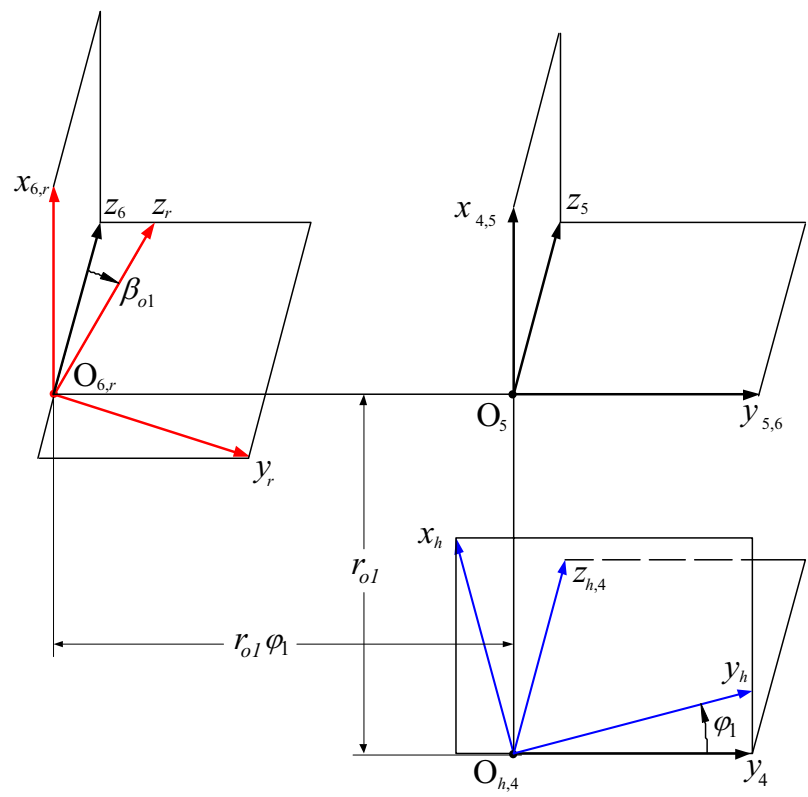

Figure 2. Coordinate systems for generation surface of the VPA hob.

\section{General mathematical model for generating the surface of grinding worm on a universal machine}

Theoretically, for grinding the surface of VPA hob, a general coordinate systems is established for obtaining a corresponding grinding worm from the VPA hob as shown in Fig. 3. Wherein the coordinate systems $S_{w}\left(x_{w}, y_{w}, z_{w}\right)$ and $S_{h}\left(x_{h}, y_{h}, z_{h}\right)$ are rigidly connected to the grinding worm and hob, respectively, while the coordinate system $S_{d}\left(x_{d}, y_{d}, z_{d}\right)$ is rigidly connected to the frame of grinding machine, and $S_{a}\left(x_{a}, y_{a}, z_{a}\right)$, $S_{b}\left(x_{b}, y_{b}, z_{b}\right)$ and $S_{c}\left(x_{c}, y_{c}, z_{c}\right)$ are auxiliary coordinate systems for simplifying the coordinate transformation. The crossed angle $\gamma_{o}$ of the rotaing axes of hob and grinding worm is usually a machine-tool setting. According to Fig. 3, by applying the homogeneous coordinate transformation from $S_{h}$ to $S_{w}$, the locus and unit normal vector of grinding worm 
surface can be represented in coordinate system $S_{w}$ as follows:

$$
\begin{aligned}
& \mathbf{r}_{w}\left(u_{1}, v_{1}, \varphi_{1}, \phi_{h}, l_{z}\right)=\mathbf{M}_{w h}\left(\phi_{h}, l_{z}\right) \cdot \mathbf{r}_{h}\left(u_{1}, v_{1}, \varphi_{1}\right), \\
& \mathbf{n}_{w}\left(u_{1}, v_{1}, \varphi_{1}, \phi_{h}, l_{z}\right)=\mathbf{L}_{w h}\left(\phi_{h}, l_{z}\right) \cdot \mathbf{n}_{h}\left(u_{1}, v_{1}, \varphi_{1}\right),
\end{aligned}
$$

where

$$
\begin{aligned}
& \mathbf{M}_{w h}\left(\phi_{h}, l_{z}\right)=\mathbf{M}_{w c} \cdot \mathbf{M}_{c h}\left(\phi_{h}, l_{z}\right)= \\
& {\left[\begin{array}{cccc}
-\cos \phi_{w} & \sin \phi_{w} \cos \gamma_{o} & \sin \phi_{w} \sin \gamma_{o} & 0 \\
-\sin \phi_{w} & -\cos \gamma_{o} \cos \phi_{w} & -\cos \phi_{w} \sin \gamma_{o} & 0 \\
0 & -\sin \gamma_{o} & \cos \gamma_{o} & 0 \\
0 & 0 & 0 & 1
\end{array}\right] \cdot\left[\begin{array}{cccc}
\cos \phi_{h} & -\sin \phi_{h} & 0 & -E_{o} \\
\sin \phi_{h} & \cos \phi_{h} & 0 & 0 \\
0 & 0 & 1 & -l_{z} \\
0 & 0 & 0 & 1
\end{array}\right] .}
\end{aligned}
$$

and the transformation matrix $\mathbf{L}_{w h}$ is the sub-matrix of $\mathbf{M}_{w h}$ by deleting the last column and row, $E_{o}$ is the center distance between grinding worm and hob, $l_{z}$ is axis feed of the grinding worm, $\phi_{h}$ and $\phi_{w}$ are rotation angles of hob and grinding worm, respectively. The relation between rotation angle of the grinding worm, $\phi_{w}$, and hob, $\phi_{h}$, is defined as

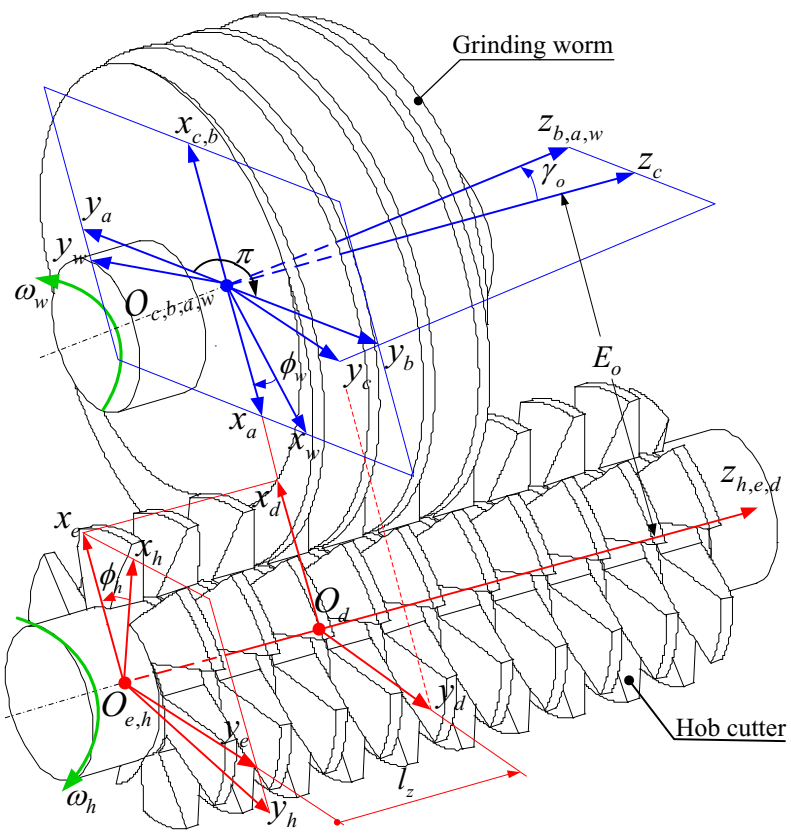

Figure 3. General coordinate systems for grinding the hob cutter with a grinding worm.

$$
\phi_{w}=\frac{N_{2}}{N_{1}} \phi_{h},
$$

where symbols $N_{2}$ and $N_{1}$ indicate the number of teeth of the hob and grinding worm, respectively.

Then, the tooth surface and unit normal vector of grinding worm can be determined by using Eqs. (6) and (7) combined with the following equations of meshing. There are two independent kinematic parameters $l_{z}$ and $\phi_{h}$ needed for grinding the helical surface of hob.
Therefore, there are two equations of meshing between the hob and the grinding worm as follows:

$$
\begin{aligned}
& f_{2}\left(u_{1}, v_{1}, \varphi_{1}, \phi_{h}, l_{z}\right)=\mathbf{n}_{w}{ }^{T} \cdot \partial_{\varphi_{h}}\left[x_{w}, y_{w}, \mathrm{z}_{w}\right]=0, \\
& f_{3}\left(u_{1}, v_{1}, \varphi_{1}, \phi_{h}, l_{z}\right)=\mathbf{n}_{w}{ }^{T} \cdot \partial_{l_{h}}\left[x_{w}, y_{w}, \mathrm{z}_{w}\right]=0,
\end{aligned}
$$

\section{Mathematical model for the generation of VPA hob on a CNC worm grinding machine}

Fig. 4 presents the 3-D model and seven motion axes of a worm grinding machine. Wherein four axes $A_{1}, X_{1}, Y_{1}$ and $Z_{2}$, are used for setting tool: $A_{1}$ is the rotary axis of the worm-head that uses for setting crossed angle between grinding worm and hob, $X_{1}$ is the horizontal axis respect to the VPA hob that uses for setting center distance between grinding worm and hob, $Y_{1}$ is the vertical axis for setting position of grinding worm and $Z_{2}$ for setting position of grinding hob. And three axes $B_{1}, B_{2}$ and $Z_{1}$ are are used for generating tooth surfaces of the VPA hob: $B_{1}$ is the rotary axis of the grinding worm spindle, $B_{2}$ is the rotary axis of workpiece spindle and $Z_{1}$ is the tangential axis of griding worm. Fig. 5 shows coordinate systems of the $\mathrm{CNC}$ worm grinding machine. By applying the homogeneous coordinate transformation matrix equation, transforming from coordinate system $S_{1}$ to $S_{2}$, the locus of the grinding worm, $\mathbf{r}_{1}$, and the unit normal vector, $\mathbf{n}_{1}$, in the coordinate system $S_{1}$, can be represented in coordinate system $S_{2}$ as follows:

$$
\begin{aligned}
\mathbf{r}_{2}\left(C_{x}, C_{y}, C_{z}, \phi_{1}, \phi_{2}\right) & =\mathbf{M}_{21}\left(C_{x}, C_{y}, C_{z}, \phi_{1}, \phi_{2}\right) \cdot \mathbf{r}_{1} \\
\mathbf{n}_{2}\left(C_{x}, C_{y}, C_{z}, \phi_{1}, \phi_{2}\right) & =\mathbf{L}_{21}\left(C_{x}, C_{y}, C_{z}, \phi_{1}, \phi_{2}\right) \cdot \mathbf{n}_{1}, \\
\mathbf{M}_{21}\left(C_{x}, C_{y}, C_{z}, \phi_{1}, \phi_{2}\right) & =\mathbf{M}_{2 m}\left(C_{x}, C_{y}, \phi_{2}\right) \cdot \mathbf{M}_{m 1}\left(C_{z}, \phi_{1}\right) .
\end{aligned}
$$

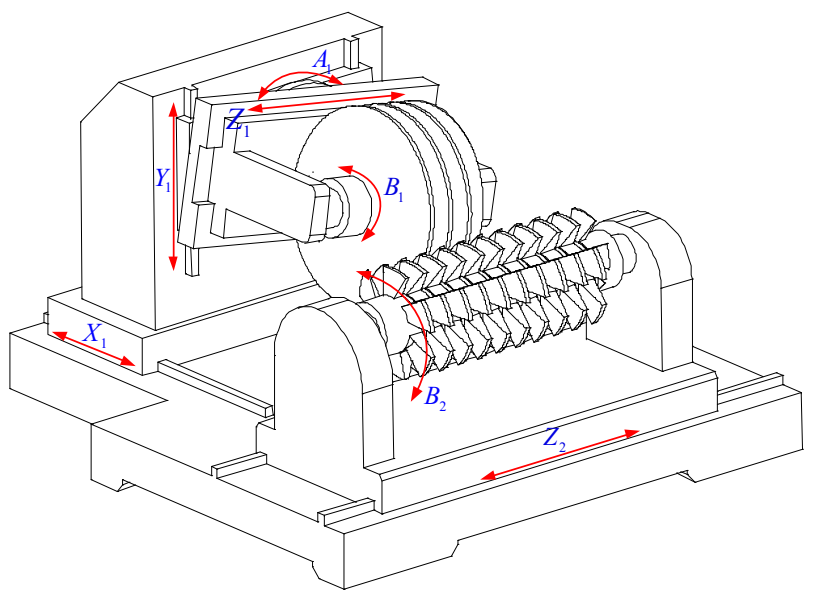

Figure 4. 3-D model of a CNC worm grinding machine. 


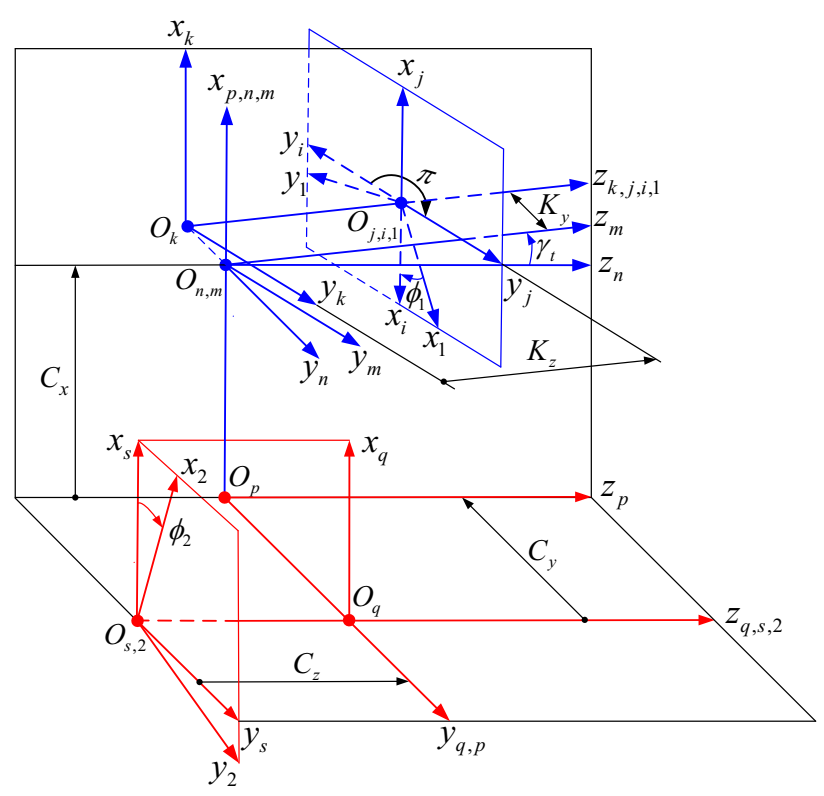

Figure 5. Coordinate system of a $\mathrm{CNC}$ worm grinding machine.

where

$\mathbf{M}_{2 m}=\left[\begin{array}{cccc}\cos \phi_{2} & \sin \phi_{2} & 0 & C_{x} \cos \phi_{2}-C_{y} \sin \phi_{2} \\ -\sin \phi_{2} & \cos \phi_{2} & 0 & -C_{y} \cos \phi_{2}-C_{x} \sin \phi_{2} \\ 0 & 0 & 1 & K_{z} \\ 0 & 0 & 0 & 1\end{array}\right]$,

$\mathbf{M}_{m 1}=\left[\begin{array}{cccc}-\cos \phi_{1} & -\sin \phi_{1} & 0 & 0 \\ \cos \gamma_{t} \sin \phi_{1} & -\cos \gamma_{t} \cos \phi_{1} & -\sin \gamma_{t} & -K_{y} \cos \gamma_{t}-C_{z} \sin \gamma_{t} \\ \sin \phi_{1} \sin \gamma_{t} & -\cos \phi_{1} \sin \gamma_{t} & \cos \gamma_{t} & C_{z} \cos \gamma_{t}-K_{y} \sin \gamma_{t} \\ 0 & 0 & 0 & 1\end{array}\right]$, and $\mathbf{L}_{21}$ is the upper-left $(3 \times 3)$ sub-matrix of the $(4 \times 4)$ homogeneous coordinate transformation matrix $\mathbf{M}_{21}$.

The spatial relative position of the grinding worm with respect to the VPA hob should be the same whether the VPA hob is ground on an universal or a CNC worm grinding machine. Therefore, the Eq. (8) and Eq. (14) should be satisfied the following relation:

$$
\mathbf{M}_{21}\left(C_{x}, C_{y}, C_{z}, \phi_{1}, \phi_{2}\right)=\mathbf{M}_{h w}\left(\phi_{h}, l_{z}\right)=\mathbf{M}_{w h}^{-1}\left(\phi_{h}, l_{z}\right) .
$$

Assuming that the rotational angle of the grinding worm and hob on the universal machine is the same as on the $\mathrm{CNC}$ machine as follows:

$$
\left\{\begin{array}{l}
\phi_{1}\left(\phi_{h}, l_{z}\right)=\frac{N_{2}}{N_{1}} \phi_{h} \\
\phi_{2}\left(\phi_{h}, l_{z}\right)=\phi_{h} \\
\gamma_{t}\left(\phi_{h}, l_{z}\right)=\gamma_{o}
\end{array} .\right.
$$

Combining the translation vectors in Eq. (17) with the above results yields the following solution for the translational positions:

$$
\left\{\begin{array}{l}
C_{x}\left(\phi_{h}, l_{z}\right)=E_{o} \\
C_{y}\left(\phi_{h}, l_{z}\right)=-K_{y} \cos \gamma_{o}-K_{z} \sin \gamma_{o} \\
C_{z}\left(\phi_{h}, l_{z}\right)=l_{z}-K_{y} \sin \gamma_{o}-K_{z} \cos \gamma_{o}
\end{array} .\right.
$$

The rotating angle of hob, $\phi_{2}$, is set as a function of grinding worm's rotation angle, $\phi_{1}$, and grinding worm's traverse feed, $C_{z}$ as follows:

$$
\phi_{2}=\frac{N_{1}}{N_{2}} \phi_{1}+\frac{\cot \lambda_{o 2}}{r_{o 2}} C_{z}+a \phi_{1} C_{z} .
$$

where $\lambda_{o 2}$ is the lead angle of hob, $\lambda_{o 2}=\pi / 2-\beta_{o 2}$ and $a$ is the control coefficient for rotational axis of hob.

\section{Numerical example}

The purpose of this example is to verify the merits of proposed worm grinding method. The basic data of the hob and the grinding worm are given in Table 1 . The

\begin{tabular}{|c|c|}
\hline Items & Value \\
\hline \multicolumn{2}{|l|}{ Hob data } \\
\hline Number teeth of hob $\left(N_{2}\right)$ & 1 \\
\hline Normal module $\left(m_{p n}\right)$ & 2 \\
\hline Normal circular-tooth thickness of hob $\left(s_{o n}\right)$ & $3.14 \mathrm{~mm}$ \\
\hline Normal pressure angle $\left(\alpha_{o n}\right)$ & $20.0^{\circ}$ \\
\hline Helix angle of hob $\left(\beta_{o 2}\right)$ & $87.8^{\circ}$ R.H. \\
\hline Lead angle of hob $\left(\lambda_{o 2}\right)$ & $2.2^{\circ}$ \\
\hline Hob operating radius $\left(r_{o 2}\right)$ & $26.05 \mathrm{~mm}$ \\
\hline Hob form radius $\left(r_{f 2}\right)$ & $24.21 \mathrm{~mm}$ \\
\hline Hob outer radius $\left(r_{a 2}\right)$ & $28.05 \mathrm{~mm}$ \\
\hline Hob length $\left(F_{w 2}\right)$ & $100 \mathrm{~mm}$ \\
\hline \multicolumn{2}{|l|}{ Grinding worm data } \\
\hline Number of starts of grinding worm $\left(N_{1}\right)$ & 1 \\
\hline Number of threads of grinding worm $\left(N_{T}\right)$ & 3 \\
\hline Grinding worm operating radius $\left(r_{o 1}\right)$ & $41.0 \mathrm{~mm}$ \\
\hline Helix angle of grinding worm $\left(\beta_{o 1}\right)$ & $88.8^{\circ}$ R.H. \\
\hline Lead angle of grinding worm $\left(\lambda_{o 1}\right)$ & $1.2^{\circ}$ L.H. \\
\hline \multicolumn{2}{|l|}{ Grinding process data } \\
\hline Center distance between worm and hob $\left(E_{o}\right)$ & $87.05 \mathrm{~mm}$ \\
\hline Crossed angle between worm and hob $\left(\gamma_{o}\right)$ & $1.0^{\circ}$ \\
\hline Control coefficient $(a)$ & $-4.5 \times 10^{-5}$ \\
\hline VPA coefficient $(b)$ & $5.0 \times 10^{-6}$ \\
\hline Machine constant $\left(K_{y}\right)$ & $5.0 \mathrm{~mm}$ \\
\hline Machine constant $\left(K_{z}\right)$ & $15.0 \mathrm{~mm}$ \\
\hline
\end{tabular}
setup data of grinding machine is calculated according to the basic meshing conditions as illustrated in Ref. [1].

Table 1. Basic data of the hob and the grinding worm. 
The Topography of the theoretical VPA hob surface and proposed VPA hob surface generated by grinding worm are shown in Figs. 6-7, respectively. The maximum deviation of the tooth flank of the proposed VPA hob surface, $M_{d}=3.26 \mu \mathrm{m}$, is so small and it is approach to that of the theoretical VPA hob surface, $M_{d}=1.35 \mu \mathrm{m}$. And the ratio of deviation of the proposed VPA hob surface, $R_{d}=0.82$, is also approach to that of the theoretical VPA hob surface, $R_{d}=0.91$. It reveals that the proposed VPA hob surface generated by grinding worm fits well with the theoretical VPA hob surface, as shown in Figs. 6-7.

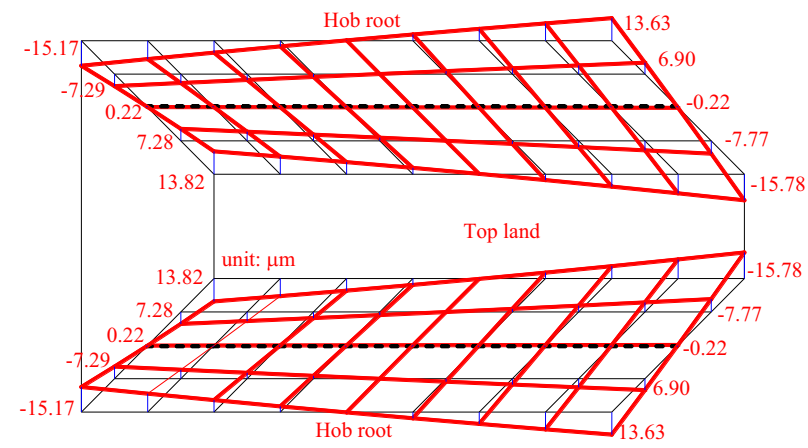

Figure 6. Topography of the theoretical VPA hob surface (maximum deviation of the tooth flank $M_{d}=1.35 \mu \mathrm{m}$ and ratio of deviation $R_{d}=0.91$ ).

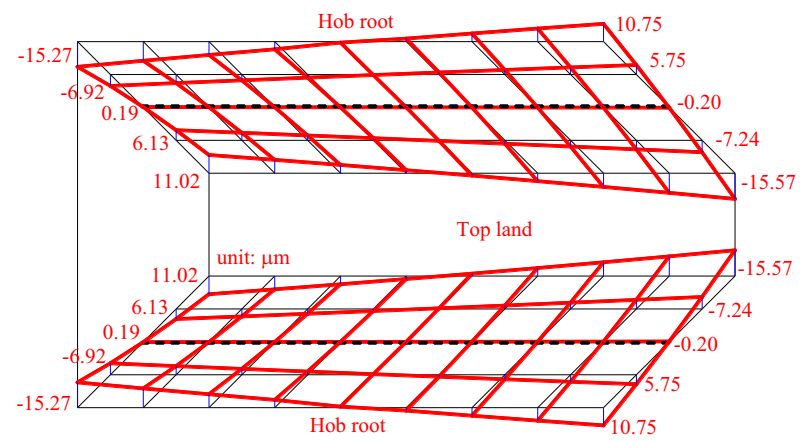

Figure 7. Topography of the proposed VPA hob surface generated by grinding worm (maximum deviation of the tooth flank $M_{d}=3.26 \mu m$ and ratio of deviation $R_{d}=0.82$ ).

\section{Conclusion}

In this paper, a new method for grinding the VPA hob with a grinding worm on a CNC machine is proposed by controlling rotational motion of hob axis (Eq. (20)). The mathematical models for generating helical surfaces of the theoretical VPA hob and the proposed VPA hob are established. The numerical result shows that the proposed VPA hob surface generated by the grinding worm can fits well with the theoretical VPA hob surface.

\section{Acknowledgment}

The authors are grateful to the Ministry of Science and Technology in Taiwan (R.O.C.) for financial supports from two projects (project no.: MOST 104-2221-E-008007 and MOST 104-2221-E-035-087).

\section{References}

1. F.L. Litvin, A. Fuentes, Gear Geometry and Applied Theory, $2^{\text {nd }}$ ed., Cambridge University Press, Cambridge, UK (2004)

2. W. Wirz, Process for machining a worm-type workpiece with a worm-type tool, Patent No. US 4475319 A (1984)

3. W. Wirz, Method, tool and device for the profiling of grinding worms for continuous gear grinding, Patent No. US5954568 A (1999)

4. W. Wirz, Topological profiling of grinding worms for continuous generating grinding of gear teeth, Patent No. US6386953 B1 (2002)

5. W. Thyssen, Grinding worm for the continuous generating grinding of gear wheels, Patent No. US 6257963 B1 (2000)

6. R. Jankowski, Profiling methods for generation of modified grinding worms, Patent No. US 6077150 A (2000)

7. R. Jankowski, Method for flexible profiling of grinding worms, Patent No. US 6012972 A (2000)

8. H.J. Stadtfeld, E.G. Mundt, Threaded grinding wheel and method of dressing, Patent No. US 6217409 B1 (2001)

9. A. Türich, Producing profile and lead modifications in threaded wheel and profile grinding, Gear Technology, 27(1), 54-62 (2010)

10. F. Klocke, M. Brumm, J. Reimann, Continuous Generating Gear Grinding: A report on new possibilities in the process design and analysis of continuous generating gear grinding, Gear Solutions, 31-44 (2012)

11. F. Klocke, M. Brumm, J. Reimann, Modeling of Surface Zone Influences in Generating Gear Grinding, Procedia CIRP, 8, 21-26 (2013)

12. B. Denkena, J. Köhler, A. Schindler, S. Woiwode, Continuous Generating Gear Grinding-Material engagement in gear tooth root machining, Mechanism and Machine Theory, 81, 11-20 (2014)

13. M. Ophey, J. Reimann, Prediction of Surface Zone Changes in Generating Gear Grinding, Gear Technology, 52-59 (2015)

14. V.T. Tran, R.H. Hsu, C.B. Tsay, A Novel Finish Hobbing Methodology for Longitudinal Crowning of a Helical Gear with Twist-Free Tooth Flanks by Using Dual-Lead Hob Cutters, ASME International Mechanical Engineering Congress \& Exposition, Montreal, Quebec, Canada. Paper No. IMECE201436149, 1-9 (2014)

15. V.T. Tran, R.H. Hsu, C.B. Tsay, Tooth Contact Analysis for a Double-Crowned Involute Helical Gear with Twist-Free Tooth Flanks Generated by Dual-Lead Hob Cutters, ASME Journal of Mechanical Design, 137, 1-11 (2015) 